regarding $\mathrm{JH}, \mathrm{P}, \mathrm{CT}$ and RSI were recorded concurrently via the OptoJump system (Microgate, Bolzano, Italy). LESS trials were scored independently by the authors. Statistical analyses were used to confirm inter and intra-rater reliability. Data was tested for normality and one-way factorial MANOVA was used to assess between group differences $(p<0.05)$. Ethical approval was granted by the University's Ethics Committee. Intra-class Correlation Coefficients (ICC) demonstrated excellent intra (0.96) and inter (0.94) rater reliability for the LESS in the current study. Six participants produced LESS scores deemed excellent to good (score $\geq 6$ ), 26 participants produced scores deemed moderate to poor (score $\leq 5$ ). Participants with moderate to poor LESS scores produced significantly greater P $\quad(p=0.038)$, RSI $(p=0.016)$ and lower CT $(p=0.002)$, there was no significant difference in $\mathrm{JH}$ $(p=0.842)$ between participants scoring excellent to good and moderate to poor. The current study reports excellent intra and inter rater reliability for the LESS, supporting its use as a clinical assessment tool in an elite rugby union populations. The majority of players presented with moderate to poor LESS scores, therefore landing biomechanics may need to be improved in this population. Participants scoring moderate to poor in the LESS recorded significantly higher P, CT and RSI but not JH. This suggests participants with high risk landing biomechanics may also produce higher performance measures, but these do not result in improved outcome performance such as jump height.

\section{SUNDAY STRATEGY AFFECTS MATCH DAY +2 SALIVARY CORTISOL AND SIGA RESPONSE FOLLOWING SATURDAY MATCHES THROUGH AN ENGLISH CHAMPIONSHIP FOOTBALL CLUB SEASON}

1) Dunbar, ${ }^{2} \mathrm{M}$ King, ${ }^{2} \mathrm{C}$ Haslam, ${ }^{3,4} \mathrm{~A}$ Nevill, ${ }^{3,5} \mathrm{G}$ Warrington. ${ }^{1}$ Soma Bioscience Limited, Wallingford, UK; ${ }^{2}$ Brentford Football Club, Brentford, UK; ${ }^{3}$ Department of Physical Education and Sport Sciences, University of Limerick, Ireland; ${ }^{4}$ Institute of Sport and Human Science, University of Wolverhampton, UK; ${ }^{5}$ Health Research Institute, University of Limerick, Ireland

\subsection{6/bjsports-2018-ISSSMC2018.7}

The measurement of salivary biomarkers has become popular in professional sport, in an attempt to monitor the stress responses associated with training, competition, and other related lifestyle factors. Using a 'Point of Care' platform, giving results within minutes of sample collection in the professional football environment, the aim is to provide coaching staff with 'readiness to train' data relating to individual players or the squad as a whole. At this club, after a Saturday match where there is no game until the following Saturday, players do not report to training until Monday Morning. However, if the next game is midweek, players are required to report to training on Sunday morning for recovery protocols. Salivary samples were collected for evaluation of sIgA and cortisol from 26 players (age $24.1 \pm 2.9$ y, body mass 78.7 $\pm 6.5 \mathrm{~kg}$, stature $1.81 \pm 0.07$ ) in a Championship football club squad at 09:00 on the Monday following a Saturday Match throughout the 2017-2018 season. In total 19 time-points were analysed; 11 where players reported in on a Sunday and 8 where players had the Sunday off from training post-match. All saliva samples were analysed at the training ground using Soma dual sIgA/Cortisol LFDs read with a Soma Cube LFD Reader to give rapid quantitative values for sIgA and cortisol.
sIgA was seen to be variable, both within (CV 53.8\%) and between players (CV 62.1\%), as was the cortisol response (within CV 53.2\% and between players CV 65.3\%). MultiLevel regression analysis revealed a highly significant quadratic effect in sIgA due to 'weeks' throughout the season (the intercept at zero weeks was $187 \mu \mathrm{g} / \mathrm{mL}$ initially increasing at the early weeks, peaking mid-season then declining significantly towards the end of the season). Where players reported to training on Sunday, the Monday sIgA response was $60 \mu \mathrm{g} / \mathrm{mL}$ lower than when Sundays were spent at home. The Model for cortisol response showed a highly significant linear increase throughout the season. However, the impact of reporting for recovery training on Sunday had a significant impact on Monday cortisol, changing from 4.9 (0.8) nM when they did report in on Sunday to 9.6 (0.6) nM when they did not. Such biomarker responses may have important implications and practical applications for the planning of recovery strategies for subsequent professional football matches.

\section{THE SALBUTAMOL PASSPORT: HOW TO RULE OUT AN ADVERSE ANALYTICAL FINDING FROM SERIAL URINE TESTS}

${ }^{1}$ Daren Austin, ${ }^{2}$ Morten Hostrup, ${ }^{3}$ Sheila M Bird. 'Senior Fellow and Senior Director, Clinical Pharmacology, GlaxoSmithKline; '2Associate Professor, Section of Integrative Physiology, Department of Nutrition, Exercise and Sports, University of Copenhagen; ${ }^{3}$ MRC Biostatistics Unit, University of Cambridge Institute for Public Health

\subsection{6/bjsports-2018-ISSSMC2018.8}

Salbutamol is used widely by elite athletes in treatment of asthma and related conditions, such as exercise-induced bronchoconstriction. In competitive sport, salbutamol is permitted by inhalation at doses up to $1600 \mu \mathrm{g} / \mathrm{day}$, not to exceed $800 \mu \mathrm{g}$ in any 12 hour period. WADA has established a urinary salbutamol Decision Limit for a presumed adverse analytical finding (AAF) of $1200 \mathrm{ng} / \mathrm{mL}$. Urine salbutamol levels greater than this are deemed to be a result of prohibited use or excessive supratherapeutic inhalation. Studies have shown that under dehydrated conditions, exercise increases the risk of exceeding the Decision Limit after single inhaled doses of $1600 \mu \mathrm{g}$. One means of explaining an AAF is a Pharmacokinetic (PK) Study to establish whether an athlete is an outlier with higher urine concentrations than typical. However, a recent salbutamol case from the 2017 Vuelta a España, deemed it impractical and impossible to conduct a valid PK study capable of recreating the complex conditions of an athlete competing in such an event. Another means of explanation is the 'Salbutamol Passport'. This is a statistical model derived from serial test data obtained from an event during periods of stable salbutamol use. After adjustment for dose and dosing frequency, the Salbutamol Passport can be used to predict an expected concentration range for days when an athlete might increase their salbutamol intake due to worsening symptoms. Since the number of tests during stable use is typically small, the method uses propagation of uncertainty in mean and variance of the log-transformed salbutamol concentrations to derive expected bands for any dose and frequency of interest. The method is demonstrated with data from a cyclist competing in the 2007 Giro d'Italia. The athlete provided four salbutamol tests whilst taking $400 \mathrm{ug} /$ day with geometric mean $461 \mathrm{ng} / \mathrm{mL}$ (95\% CL: 352-604) and one test of 
$1352 \mathrm{ng} / \mathrm{mL}$ after allegedly increasing his dose to $700 \mathrm{ug} / \mathrm{day}$. Extrapolating the $400 \mathrm{ug} /$ day data to $700 \mathrm{ug} / \mathrm{day}$, and assuming an increase from twice-daily to three-times daily dosing, the Salbutamol Passport prediction is $1050 \mathrm{ng} / \mathrm{mL}$ (95\%PI: 802-1375). Since the observed test falls within the 95\% prediction interval, the result would not constitute an AAF. This conclusion is unchanged after adjustment of salbutamol levels to a urine specific gravity of 1.020 .

\section{DIETARY PRACTICES OF PHYSICALLY ACTIVE 9-12-YEAR OLD CHILDREN, PHYSICAL ACTIVITY AND NUTRITIONAL KNOWLEDGE OF THEIR PARENTS}

EC Wauchope. Department of Sociology (Sport and Exercise Sciences), Durham University, UK

\subsection{6/bjsports-2018-ISSSMC2018.9}

Healthy eating through a balanced diet is fundamental to good health. Active children are of interest given the energy demands of preparing for athletic performance or competition alongside normal growth and development. There is minimal research into the requirements of preadolescent athletes. It is unknown whether findings and recommendations in adult athletes can be extrapolated to their junior counterparts. Active 9-12 year old children $(n=18)$ from the North East of England completed three-day estimated weight food diaries, like the National Diet and Nutrition Survey (2016). Secondary outcomes determined self-reported physical activity level using the Physical Activity Questionnaire for Children, and parental scores of the General Nutrition Knowledge Questionnaire. Daily averages of energy, fibre, macronutrients (protein, carbohydrates and fat) and key micronutrients (Vitamin C, Vitamin $\mathrm{D}$, iron, calcium and sodium) were analysed using McCance and Widdowson's Composition of Foods (2015). To contextualise the nutrition findings, data were compared to national recommendations and the National Diet and Nutrition Survey (2016). Energy intake (7.2 MJ/day) was higher than for children nationally but lower than recommended. This was principally driven by low intake of carbohydrates $(234 \mathrm{~g} /$ day $)$ and fat $(62 \mathrm{~g} /$ day $)$. Intake of protein was comparatively high $(69 \mathrm{~g} /$ day) and total fibre $(9 \mathrm{~g} /$ day $)$ low. Intake of Vitamin C (112 mg/day), iron (13 mg/day) and calcium (732 mg/day) was sufficient and Vitamin D $(2.5 \mu \mathrm{g} /$ day $)$ low. Secondary outcomes analysed associations between daily nutritional values and child physical activity and parent nutritional knowledge scores using the Pearson correlation co-efficient. There were no relationships $(p>0.05)$ between energy intake and either of these variables. Findings suggested that active 9-12 year old children consume more energy than indicated by UK datasets, but consume less energy than national recommendations for gender and age. Principally this was driven by low intake of carbohydrate and fat. No significant relationships were apparent between energy intake and physical activity level, or energy intake and parent nutrition knowledge score. These findings are important to better-understand and support nutritional needs of active children.

\section{COMPARING THREE ANALYSIS METHODS FOR KINEMATIC DATA EVALUATION}

$\mathrm{S}$ Alizadeh, K Mattes. Department of Movement and Training Sciences, University of Hamburg, Hamburg, Germany

\subsection{6/bjsports-2018-ISSSMC2018.10}

The purpose of this study was to compare three different methods of data analysis: conventional, Statistical Non-Parametric Mapping (SnPM) and Functional Data Analysis (FDA) for a series of kinematic data acquired from soccer players from their dominant and non-dominant leg at the late swing phase while sprinting. Kinematic data was acquired via Vicon motion analysis system at $200 \mathrm{~Hz}$. For conventional computation, local maximum, mean and minimum values were extracted for further analysis. A two-tailed dependent t-test was implemented for statistical analysis for the conventional method. A two-tailed SnPM and a one-tailed FDA permutation test was performed on the same data $(\alpha=0.05)$. All the trials where filtered using zero-lag 4th order Butterworth filter. The cutoff frequency $(13 \mathrm{~Hz})$ was calculated using the optimum cutoff frequency formula. Late-swing phase was defined as the moment where the knee started to extend from its flexed position in the 2nd half of swing phase till foot contact. The conventional results show there was a difference in the mean knee angle in the sagittal plane between the dominant $\left(\theta^{\circ}=73.0\right)$ and non-dominant leg $\left(\theta^{\circ}=74.7\right)$ $(\mathrm{p}=0.04)$. Both SnPM (\% late swing phase found significant $=28.9 \%-86.1 \%, \quad \mathrm{t}$-value $=2.52, \mathrm{p}<0.001)$ and FDA $(\%$ late swing phase found significant $=28.1 \%-87.7 \%$, tvalue $=2.47-2.55, \mathrm{p}<0.001$ ) exhibited a significant difference between the knee angle of the dominant and non-dominant leg at the late swing phase. However, the SnPM demonstrated reliability in producing result while FDA presented a fluctuation, although minor, in the calculation of $p$ value. SnPM would produce more reliable and comprehensive result in comparison to FDA and conventional methods. SnPM enables scientist to identify deviation in movement pattern in soccer players which is not limited to a single point in time and can identify deficiency in movement pattern which may hinder performance or lead to injury. 